\title{
Erratum to: Allometric equations for estimating aboveground biomass of Coffea arabica L. grown in the Rift Valley escarpment of Ethiopia
}

\author{
Mesele Negash • Mike Starr • Markku Kanninen • \\ Leakemaraiam Berhe
}

Published online: 14 August 2013

(C) Springer Science+Business Media Dordrecht 2013

\section{Erratum to: Agroforest Syst (2013) 87:953-966 DOI 10.1007/s10457-013-9611-3}

In the original publication of the article, the data in Table 2 "Summary statistics of biometric parameters of the harvested coffee plant samples $(n=31)$ " the crown area $\left(\right.$ Crown $\left.n_{\text {area }}\right)$ calculated for 31 sample trees based on crown width $\left(\right.$ Crown $\left._{\text {wid }}\right)$ has been published incorrectly.

The values of Crown $n_{\text {area }}$ mean, minimum, maximum and SD are corrected and the complete version of Table 2 is provided below.

The online version of the original article can be found under doi:10.1007/s10457-013-9611-3.

M. Negash $(\bowtie) \cdot$ M. Kanninen

Department of Forest Sciences, Viikki Tropical Resources Institute, University of Helsinki, Latakartanonkarri 7,

P.O. Box 27, 00014 Helsinki, Finland

e-mail: mesele.tesemma@helsinki.fi;

kelemuamesele@yahoo.com

Present Address:

M. Negash · L. Berhe

Wondo Genet College of Forestry and Natural Resources, Hawassa University, P.O. Box 128, Shashemene, Ethiopia

M. Starr

Department of Forest Sciences, University of Helsinki, Latokartanonkaari 7, P.O. Box 27, 00014 Helsinki,

Finland
Table 2 Summary statistics of biometric parameters of the harvested coffee plant samples $(n=31)$

\begin{tabular}{llrlcl}
\hline Parameters & Unit & Mean & Minimum & Maximum & SD \\
\hline$d$ & $\mathrm{~cm}$ & 9.57 & 3.00 & 18.30 & 3.22 \\
$d_{40}$ & $\mathrm{~cm}$ & 11.90 & 3.80 & 22.80 & 3.76 \\
$h$ & $\mathrm{~m}$ & 5.59 & 4.10 & 7.00 & 0.82 \\
$h_{\text {dom }}$ & $\mathrm{m}$ & 5.80 & 4.10 & 7.00 & 0.85 \\
Crown $_{\text {wid }}$ & $\mathrm{m}$ & 3.04 & 1.30 & 4.30 & 0.70 \\
Crown $_{\mathrm{h}}$ & $\mathrm{m}$ & 3.34 & 2.00 & 4.50 & 0.51 \\
Crown $_{\text {area }}$ & $\mathrm{m}^{2}$ & 7.26 & 1.23 & 14.19 & 3.09 \\
\hline
\end{tabular}

$d$ diameter at breast height, $d_{40}$ stump diameter at $40 \mathrm{~cm}$ height, $h$ total height, $h_{\text {dom }}$ dominant height, Crown wid $_{\text {crown }}$ width, Crown $n_{h}$ crown height, Crown area crown area 